\title{
Negative priming by rotated objects
}

\author{
JANICE E. MURRAY \\ University of Otago, Dunedin, New Zealand
}

\begin{abstract}
Previous work has found that repeated exposure to ignored rotated objects is insufficient to allow the formation of orientation-invariant representations (Murray, 1995b). In this study, the negative priming paradigm was used to examine whether the identity of ignored rotated objects was encoded. Subjects were briefly presented with a prime followed by a probe display. One of the two overlapping drawings of objects in each display was selected for further processing, and the other was ignored. In one condition, the ignored objects were upright; in another, they were rotated $240^{\circ}$; and in a final condition, subjects repeatedly named the $240^{\circ}$ objects prior to experiencing them as ignored objects in the priming task. Naming latency for the attended probe was slower when it was semantically related to the ignored prime in all conditions. The results suggest that unattended rotated objects are processed to a level of representation that is at least categorical.
\end{abstract}

Past research investigating the role of attention in object recognition has dealt primarily with the processing of a small set of upright objects. This work has suggested that while ignored objects are not remembered (e.g., Goldstein \& Fink, 1981; Rock \& Gutman, 1981; Tipper, 1985), they may be fully encoded and achieve a level of representation that is at least categorical (e.g., Tipper, 1985; Tipper \& Driver, 1988). Little, however, is known about what effect the withdrawal of attention has on the perceptual operations involved in recognition of objects presented at nonupright orientations.

Recently, Murray (1995b) demonstrated that allocation of attention is necessary for the formation and use of orientation-invariant representations in the identification of rotated objects. In one condition, subjects were asked to name a rotated line drawing of an object and ignore a changing letter display centered on the object. In another condition, subjects were asked to count the number of red Ts in the sequence of individually presented red or blue Ts and $\mathrm{Xs}$ and ignore the object. When subjects who had previously ignored the rotated objects over repeated presentations finally identified the objects, a robust effect of orientation was found; the time to name drawings of rotated objects increased linearly with increasing departures from the upright for orientations from $0^{\circ}$ to $120^{\circ}$. Moreover, the magnitude of the orientation effect was comparable to that observed for objects viewed and identified for the first time, suggesting that similar orientation-dependent representations and processes are used in the two identification contexts.

This marked effect of orientation for previously ignored objects was in contrast to the reduced orientation effect found in the condition where the rotated objects had been

This research was supported by grants from the New Zealand Foundation for Research, Science and Technology and the University of Otago Research Committee. I thank James Dignan for assistance with data collection. Correspondence regarding this paper may be sent to the author, Department of Psychology, University of Otago, Box 56, Dunedin, New Zealand (e-mail: jmur@rivendell.otago.ac.nz). fully attended to and named in the earlier presentations. The latter condition characterizes what is typically found in the identification of rotated objects following repeated naming. When rotated objects are viewed and identified for the first time, naming time increases as a function of degree of rotation from the upright, as described above (e.g., Jolicoeur, 1985; Maki, 1986; Murray, Jolicoeur, McMullen, \& Ingleton, 1993). This suggests the involvement of normalization processes in the initial identification of objects where a process such as mental rotation is required to maintain the spatial relations among features or parts for comparison with relations stored in orientation-dependent representations in memory (Jolicoeur, 1990). Following increased familiarity with the same objects through repeated identification, the initial effects of orientation on naming are much reduced in magnitude (e.g., Jolicoeur, 1985; Murray et al., 1993). This suggests that increased familiarity with a set of rotated objects results in the mediation of identification by orientation-invariant representations. Results further suggest that what is learned with practice is which orientation-invariant attributes or features reliably distinguish one object from others in the experienced set (Jolicoeur, 1985; Jolicoeur \& Milliken, 1989; Murray et al., 1993). The finding that this shift from orientation-dependent to orientation-invariant identification does not take place when objects have not been allocated attention indicates that at some stage in the processing of rotated objects, attention plays an important role.

One possibility suggested by the results of Murray (1995b) is that the effect of attention is felt very early in the processing of rotated objects, prior to the identification of the rotated object, and that without allocation of attentional resources, requisite orientation-dependent normalization processes which lead to identification do not operate. If attention is required for the initiation and/or execution of normalization processes, then identification of ignored objects would not occur according to this view, and orientation-invariant representations would not be formed. Consequently, subsequent naming times for previously ig- 
nored objects would show a robust orientation effect similar to that observed for objects viewed and named for the first time.

The absence of a shift to orientation-invariant identification for previously ignored objects does not necessarily demand an early selection explanation. Murray's results can be accommodated equally by considering an effect of attention that is felt later in the processing of rotated objects after identification, at the level of encoding orientationinvariant attributes. Without allocation of attention, identification processes may still operate and result in full identification of the ignored rotated objects. However, the selection, comparison, and storage processes required for the formation of orientation-invariant representations to enable subsequent orientation-free identification of rotated objects may require attention. Without attention, repeatedly ignored rotated objects would yield robust orientation effects when finally attended to and named.

The question of whether the identity of unattended rotated objects is encoded was investigated in the present experiment using the negative priming paradigm (Tipper, 1985). Tipper has shown that when an attended probe drawing is immediately preceded by an ignored prime drawing from the same semantic category, naming response to the attended probe is slower than when the drawings are unrelated (Tipper, 1985; Tipper \& Driver, 1988). This has been taken as evidence that ignored objects are processed to at least an abstract semantic level of representation. If similar priming effects can be obtained when the ignored prime is rotated, then this would suggest some encoding of object identity.

The effect of prior experience in naming the rotated objects that would later serve as ignored primes was aiso examined. Practice in naming the rotated objects prior to performing the priming task would result in the formation of orientation-invariant representations for those objects (e.g., Jolicoeur, 1985). The attentional requirements for identification based on simple orientation-invariant features may well be expected to differ from those for orientationdependent identification where the spatial relations among features are maintained (Treisman \& Gelade, 1980).

\section{METHOD}

\section{Subjects}

Eighty-four students (43 females) of the University of Otago participated as subjects. All were native speakers of English without previous experience with the stimuli, and had normal or corrected-to-normal vision. Testing for each subject was $50 \mathrm{~min}$ in duration.

\footnotetext{
Apparatus and Stimuli

The stimuli in the selective attention task were presented by a fourfield Gerbrands tachistoscope. Voiced responses were detected by a voicekey and measured by a Lafayette millisecond timer. An IBM-compatible computer with a Zenith 1492 VGA color monitor was used for stimulus preparation and presentation of stimuli in a preliminary naming task.

The stimuli were 10 line drawings of objects and animals selected from Snodgrass and Vanderwart (1980). The drawings were chosen from five semantic categories, and the related pairs were cat-dog, leg-foot, chair-table, coat-jumper (sweater), and car-plane. Stored images of the drawings were created by scanning each drawing using an HPScanjet Plus at $0^{\circ}$ and $120^{\circ}$ of clockwise rotation. Both $0^{\circ}$ and $120^{\circ}$ versions of the
}

stimuli were next converted to green line drawings, and these were used as ignored stimuli. Additionally, the $0^{\circ}$ drawings were produced as red line drawings to serve as selected stimuli. To facilitate their selection, the drawings in the red set were drawn with an extra pixel width. The drawings ranged in size from $4.3^{\circ}$ to $7.4^{\circ}$ of visual angle.

Two sets of display pairs were created by superimposing red drawings over green drawings. In the upright set, both the green and red drawings were at $0^{\circ}$. In the rotated set, the green drawings were at $120^{\circ}$ of clockwise rotation. When viewed in the tachistoscope, these $120^{\circ}$ drawings were seen as rotated $240^{\circ}$ from the upright. Both the upright and rotated sets were created identically as follows. Each red drawing was superimposed over every other green drawing except its same category member to produce 80 displays. All displays were then printed on an HP Deskjet $500 \mathrm{C}$ color printer. Twenty displays were selected to be used as probe displays, and 10 were selected for each of the priming conditions: at tended semantic, control, and ignored semantic. A final 10 displays were used for practice trials.

\section{Design}

All subjects received each of the three priming conditions. In every condition, all drawings in the prime and probe displays were unrelated to one another except as appropriate for the particular priming condition. In the attended semantic condition, the selected prime was semantically related to the selected probe. In the control condition, none of the selected or ignored drawings of the prime and probe were related to one another. Finally, in the ignored semantic condition, the ignored prime was semantically related to the selected probe. An example of a prime-probe sequence for the rotated set is shown in Figure 1. There were 20 trials in each priming condition, with each probe display presented once and each prime display presented twice. Across the 20 trials, each drawing was displayed twice as a selected drawing and twice as an ignored drawing in both the probe and prime displays of each priming condition. Stimulus presentation was randomly determined for each subject.

The subjects were randomly assigned to one of three experimental conditions. In the upright group, the orientation of the ignored prime was $0^{\circ}$. In the rotated group, the orientation of the ignored prime was $240^{\circ}$. Finally in the rotated-plus-practice group, the ignored prime was also $240^{\circ}$, but in this condition the subjects had practice naming the rotated drawings prior to the selective attention task. Subjects in this group initially named each of the 10 drawings presented one at a time in a block of trials. This block was repeated five times, with stimulus presentation random in each block. Although subjects in the rotated group repeatedly named the small set of objects in the attended condition of the priming task, this was not expected to result in the formation of orientation-invariant representations. Attended objects were always upright, and past research has demonstrated that when all objects are named initially as upright objects only, there is no shift from orientation-dependent to orientation-invariant identification (Jolicoeur \& Milliken, 1989; Murray, 1995a).

\section{Procedure}

Subjects were first shown upright versions of each of the 10 drawings for naming. To enhance the associative strength of same-category drawings, the drawings were presented in pairs corresponding to each of the five semantic categories (e.g., cat-dog).

Masking stimulus onset asynchrony (SOA) was next established for each subject for both prime and probe displays. These durations were the minimum time required to select and accurately identify the red drawing in each display. Three prime and three probe displays, each followed immediately by a 100 -msec pattern mask, were randomly presented to the subject. Stimulus duration for each display was initially set at $20 \mathrm{msec}$ and increased by $5 \mathrm{msec}$ using the method of ascending limits until the subject successfully identified the selected drawing. The longest SOA was recorded independently for primes and probes, and $5 \mathrm{msec}$ was added to each time. This resulted in a mean prime SOA of $83 \mathrm{msec}$ (range 45-155) and a mean probe SOA of $71 \mathrm{msec}$ (range 45-185).

In the experimental trials, a buttonpress by the subject began the trial with a fixation cross for $600 \mathrm{msec}$. This was followed immediately by the prime display. After the SOA appropriate for that subject, a pattern mask was presented for $100 \mathrm{msec}$. A fixation cross was then displayed for $1,100 \mathrm{msec}$. The probe display was next presented and followed finally by a pattern mask. 
PRIME

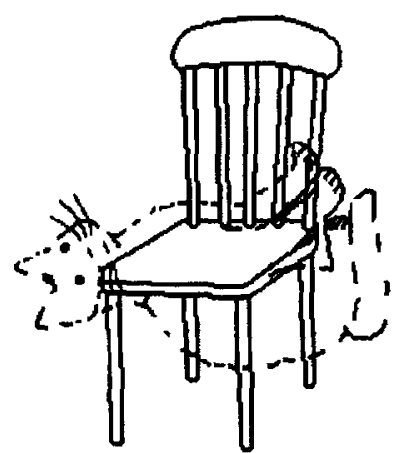

\section{PROBE}

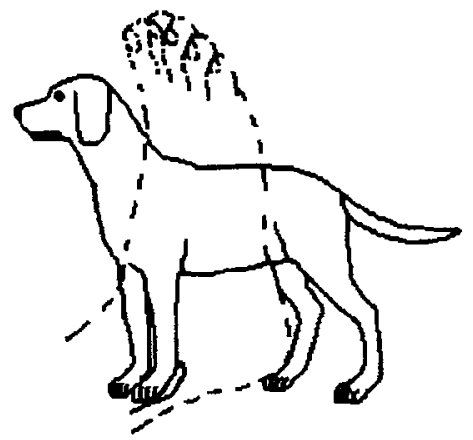

Figure 1. Examples of the prime and probe displays in the ignored rotated condition. The lines depicted as solid were red; those depicted as broken were green.

Instructions to the subjects began with an example of a red drawing superimposed over a green drawing. The subjects were told that their task was to attend to and identify the red drawing, and were informed that the green drawing was irrelevant to the task of identifying the red drawing and should be ignored. The subjects were instructed to covertly identify the red drawing of the first (prime) display for later recall. Upon presentation of the second (probe) display, the subjects were asked to name aloud the red drawing as quickly as possible, and then to recall the red drawing from the first display. It was stressed that speed in naming the second display and accuracy in identifying both display drawings were important. Each trial concluded with feedback regarding naming latency and accuracy, and the experimenter recorded both measures on the computer. Ten practice trials were given initially, and a short rest was provided halfway through the experimental trials.

On the last trial, the subjects were presented with the prime display only and asked to report the identity of the ignored green drawing. This surprise trial served as a test of the subject's adherence to the instructions to ignore the green drawing and was used to measure any awareness of the ignored stimulus.

Prior to performing the selective-attention task described above, the subjects in the rotated-plus-practice group were presented with rotated versions of the 10 drawings on a computer monitor for naming. Each black line drawing was displayed following a keypress by the subject and remained in view until the subject verbally responded. The subjects proceeded through the five blocks of preliminary naming trials without interruption.

\section{RESULTS}

No subject was able to report the ignored green drawing in the catch trial at the end of the experiment. This result is consistent with previous findings using the same methodology (Tipper, 1985; Tipper \& Driver, 1988), and suggests that subjects were successful in selection and had no awareness of the ignored stimuli.

Median response times (RTs) to the selected probe were calculated for each priming condition of each experimental group. The mean median RTs, as well as the percent error in recalling and naming the selected primes and probes respectively, are presented in Table 1.

RT negative priming effects were assessed in a mixedmodel analysis of variance (ANOVA) in which group (upright, rotated, or rotated-plus-practice) was a betweensubjects variable and priming condition (control or ignored semantic) was a within-subject variable. As is evident in
Table 1, response times to selected probes were slower in the ignored semantic condition than in the control condition $\left[F(1,81)=19.71, M S_{\mathrm{e}}=993, p<.001\right]$. There was no effect of group $(F<1)$. Most importantly, the significant negative priming effect did not vary as a function of group $(F<1)$. Individual $t$ tests confirmed the presence of a significant negative priming effect in each of the three groups ( $p<.01$ in all cases).

The analysis of prime recall errors showed that performance was less accurate in the upright group than in either of the two rotated groups $\left[F(2,81)=8.31, M S_{e}=20, p<\right.$ $.001]$. Similar results were found for probe errors; errors were greater in number for the upright group $[F(2,81)=$ $\left.12.80, M S_{\mathrm{e}}=23, p<.001\right]$. There were no other significant effects.

RT facilitatory priming effects were assessed in a mixedmodel ANOVA in which group and priming condition (control or attended semantic) were the variables. Response times were faster in the attended semantic condition than in the control condition $\left[F(1,81)=44.72, M S_{\mathrm{e}}=1,285\right.$, $p<.001]$. There were no significant differences across group $(F<1)$, and the facilitatory priming effect did not depend upon group $\left[F(2,81)=2.19, M S_{\mathrm{e}}=1,285, p<.15\right]$.

Separate analyses of prime recall and probe errors showed higher error rates in the upright group than in either of the rotated groups, for both prime $\left[F(2,81)=8.74, M S_{\mathrm{e}}=\right.$ $17, p<.001]$ and probe errors $\left[F(2,81)=18.06, M S_{\mathrm{e}}=\right.$ $17, p<.001]$. There were no other significant effects.

\section{DISCUSSION}

The results are clear. When an ignored rotated object immediately precedes a categorically related object, there is a reaction time cost in naming the second object. This negative priming effect is observed when the rotated object is experienced repeatedly in earlier naming trials, resulting in orientation-invariant identification, and also when it is experienced only as an ignored object in the priming context where identification is orientation dependent. The results indicate that there is processing of ignored rotated objects to a level of representation that is at least categorical, and are consistent with the view that the effects of withdrawal of attention are felt late in the processing of rotated objects (e.g., Tipper \& Driver, 1988). 
Table 1

Means of Median Reaction Times (in Milliseconds) to Name Probes and Percent Errors in Recalling Primes and Naming Probes

\begin{tabular}{|c|c|c|c|}
\hline & $\begin{array}{c}\text { Ignored } \\
\text { Semantic }\end{array}$ & Control & $\begin{array}{l}\text { Attended } \\
\text { Semantic }\end{array}$ \\
\hline \multicolumn{4}{|c|}{$0^{\circ}$ Ignored Prime } \\
\hline Reaction Time & 725 & 702 & 681 \\
\hline$\%$ Errors-Prime & 3.6 & 4.6 & 2.7 \\
\hline$\%$ Errors-Probe & 6.4 & 6.4 & 5.7 \\
\hline \multicolumn{4}{|c|}{$240^{\circ}$ Ignored Prime } \\
\hline Reaction Time & 733 & 705 & 660 \\
\hline$\%$ Errors-Prime & 1.3 & 0.9 & 0.7 \\
\hline$\%$ Errors-Probe & 2.1 & 2.5 & 1.3 \\
\hline \multicolumn{4}{|c|}{$240^{\circ}$ Ignored Prime With Practice } \\
\hline Reaction Time & 721 & 707 & 661 \\
\hline$\%$ Errors-Prime & 1.6 & 0.7 & 1.1 \\
\hline$\%$ Errors-Probe & 2.3 & 2.9 & 1.6 \\
\hline
\end{tabular}

If a normalization process such as mental rotation is required to align a representation of a rotated object with a stored representation for identification, then the present results in the ignored rotated condition can be interpreted as suggesting that these mental-rotation processes can operate in parallel with other processes without full allocation of attentional resources. In related work, Corballis (1986) has examined the attentional demands of mental-rotation tasks requiring normal/mirror-image judgments for letters, and obtained evidence suggesting that attentional resources are required for the setting up of processing structures necessary for mental rotation but not for its execution. Recently, VanSelst and Jolicoeur (1994) have shown that mental rotation, again in the context of normal/ mirror-image tasks. can proceed in parallel with the operations required for performance of a tone-discrimination task. These results are consistent with the view that mental rotation operates when ignored rotated objects are processed. Additional results from Murray (1995b) suggest that mental rotation does take place when objects are unattended. Murray found that performance on the letter-count task declined with the angle of rotation of the ignored object without any awareness of object identity. This effect of orientation on letter-count performance suggests that subjects briefly switch attention to either initiate or execute mental rotation in the process of identifying the ignored objects.

An alternative account of the negative priming effect for rotated objects is one in which mental rotation does not occur and objects are not fully identified. Recognition may proceed hierarchically from a broad to a more specific level (cf. Corballis, 1988). Initial processing of objects may involve extraction of information that is sufficient to place the object in a general class of stimuli (e.g., animal). This first level of identification may allow the assignment of internal axes (e.g., top and bottom), which would in turn allow mental rotation to take place. Once the object was rotated to the upright, a specific match in memory could be made and the object fully identified (e.g., cat). It is possible that identification of rotated ignored objects can occur up to the level of category alone, with further processing to fully identify the object requiring attention. Inhibition could take place at the categorical level to produce the effects observed in negative priming (Tipper, 1985).

Another possibility is that because a small set of stimuli are presented repeatedly, both as ignored stimuli and as attended prime and probe stimuli, single features may be more easily encoded and used to identify each stimulus independently of its orientation or attentional status. This explanation seems an unlikely account of the negative-priming effect for ignored rotated objects. As described previously, there is no evidence to suggest that orientation-invariant features are used to identify rotated objects either following repeated presentation of ignored rotated objects (Murray, 1995b) or following repeated naming of upright objects viewed in an upright context (Jolicoeur \& Milliken, 1989; Murray, 1995a). However, the effect of stimulus repetition may be worthy of further study.

One other aspect of the data is deserving of comment. Error rates were higher in the upright condition than in either of the rotated condi- tions for both prime and probe displays. One possible implication of this result is that when ignored objects are in a more familiar orientation, such as upright, they are more difficult to completely filter out. This effect, however, did not interact with the relation between the ignored prime and the attended probe. Also, the negative priming effect was comparable across upright and rotated conditions. Regardless of whether or not ignored objects have some interfering effect, the negative priming data indicate that all ignored objects are processed to an abstract level of representation. This finding is consistent with previous work contrasting interference and priming (Driver \& Tipper, 1989)

In conclusion, converging evidence is required to determine whether ignored objects are fully identified through a normalization process such as mental rotation. What is clear is that there is encoding of ignored rotated objects to a level that is at least categorical, and further, that this processing is insufficient to result in the formation of orientationinvariant representations (Murray, 1995b).

\section{REFERENCES}

Corballis, M. C. (1986). Is mental rotation controlled or automatic? Memory \& Cognition, 14, 124-128.

Corballis, M. C. (1988). Recognition of disoriented shapes. Psychological Review, 95, 115-123.

DrIver, J., \& TiPPER, S. P. (1989). On the nonselectivity of "selective" seeing: Contrasts between interference and priming in selective attention. Journal of Experimental Psychology: Human Perception \& Performance, 15, 307-314.

Goldstein, E. B., \& Fink, S. I. (1981). Selective attention in vision: Recognition memory for superimposed line pictures. Journal of Experimental Psychology: Human Perception \& Performance, 10, 640656.

JoLICOEUR, P. (1985). The time to name disoriented natural objects Memory \& Cognition, 13, 289-303.

JOLICOEUR, P. (1990). On the role of mental rotation and feature extraction in the identification of disoriented objects: A dual-system theory. Mind \& Language, 5, 387-410.

JolicoeUR, P., \& MILLIKEN, B. (1989). Identification of disoriented objects: Effects of context of prior presentation. Journal of Experimental Psychology: Learning, Memory, \& Cognition, 15, 200-2 l0.

MAKI, R. H. (1986). Naming and location of tops of rotated pictures. Canadian Journal of Psychology, 40, 368-387.

Murray, J. E. (1995a). Imagining and naming rotated natural objects. Psychonomic Bulletin \& Review, 2, 239-243.

MurRAY, J. E. (1995b). The role of attention in the shift from orientationdependent to orientation-invariant identification of disoriented objects. Memory \& Cognition, 23, 49-58.

Murray, J. E., Jolicoeur, P., McMullen, P. A., \& Ingleton, M. (1993). Orientation-invariant transfer of training in the identification of rotated natural objects. Memory \& Cognition, 21, 604-610.

Rock, I., \& GutMaN, D. (1981). Effect of inattention on form perception. Journal of Experimental Psychology: Human Perception \& Performance, 7, 275-285.

SNODGRASS, J. G., \& VANDERWART, M. (1980). A standardized set of 260 pictures: Norms for name agreement, image agreement, familiarity, and visual complexity. Journal of Experimental Psychology: Human Learning \& Memory, 6, 174-215.

TIPPER, S. P. (1985). The negative priming effect: Inhibitory effects of ignored primes. Quarterly Journal of Experimental Psychology, 37, 571-590.

TIPPER, S. P., \& Driver, J. (1988). Negative priming between pictures and words in a selective attention task: Evidence for semantic processing of ignored stimuli. Memory \& Cognition, 16, 64-70.

Treisman, A., \& Gelade, G. (1980). A feature-integration theory of attention. Cognitive Psychology, 14, 107-141.

VANSELST, M., \& Jolicoeur, J. (1994). Can mental rotation occur before the dual-task bottleneck? Journal of Experimental Psychology: Human Perception \& Performance, 20, 905-921.

(Manuscript received February 16, 1995; revision accepted for publication May 9, 1995.) 